\title{
Rapid synthesis of a changing evidence base during the COVID-19 pandemic: the NeoCLEAR Project
}

\author{
Megan J. Kirkley $\mathbb{D}^{1,2} \cdot$ Clyde J. Wright ${ }^{2,3}$
}

Received: 26 November 2020 / Revised: 14 January 2021 / Accepted: 17 February 2021 / Published online: 4 March 2021

(c) The Author(s), under exclusive licence to Springer Nature America, Inc. 2021

\section{Introduction}

The COVID-19 pandemic became reality in the USA in the spring of 2020, posing acute challenges to healthcare providers and administrators. The novel nature and rapid spread of SARS-CoV-19 added a layer of unprecedented complexity. Questions arose in real-time, with no evidence or literature base to assist healthcare workers in answering them. These issues were related, but not limited to managing hospital census, the need for PPE and testing supplies, potential treatment options, understanding mechanisms of spread, and identifying high risk populations. Much was (and remains) unknown about this virus, its biology, and the policies and practices that best prevent its spread.

As practitioners in a relatively young subspecialty, neonatologists are characterized by a rigorous commitment to contributing to and keeping abreast of a rapidly changing evidence base. This was critical in the early stages of the US wave of the Coronavirus-19 pandemic. Neonatologists also faced the need to respond quickly and decisively with changes in unit policies intended to protect mothers, babies, and NICU care team members. Here we report how we responded to a growing body of literature related to this global pandemic. Specifically, we report how we curated the literature, creating a centralized repository, and making it accessible to neonatologists around the world.

\section{Background}

As clinicians facing an exponential increase in the number of patients with or at risk for developing COVID-19 related

\footnotetext{
$\checkmark$ Megan J. Kirkley

Megan.kirkley@dhha.org

Denver Health Medical Center, Denver, CO, USA

2 University of Colorado School of Medicine, Aurora, CO, USA

3 Children's Hospital Colorado, Aurora, CO, USA
}

illnesses, several questions arose in real-time. We identified questions about SARS-CoV-19 that were unique to the practice of neonatology:

- Is there maternal-to-infant transmission of COVID-19?

- Who should wear masks? What type?

- What is the aerosolization risk of a routine delivery, or a neonatal intubation?

- How do infants manifest infection?

- Can a positive test in a newborn be trusted?

- Does COVID-19 have different implications for preterm or medically fragile infants?

- Is breastfeeding safe?

Not developing action plans to address these questions would leave patients and healthcare workers at risk for exposure and symptomatic disease. However, overreacting could contribute to a strain on healthcare resources and potentially negatively impact patient well-being through over-intervention. To address the need for rapid synthesis and dissemination of COVID-19 related evidence thoughtfully generated by colleagues around the world that had already experienced these acute needs, we convened NeoCLEAR: Neonatal Coronavirus Literature Evaluated and Adapted in Real-time.

\section{Structure}

The NeoCLEAR workgroup mobilized academic neonatologists in our section who faced temporary research lab suspensions and were interested in emerging COVID-19 literature. We tasked them with systematically identifying, reviewing and compiling all literature related to COVID-19 infection in pregnant women and neonates.

First, our workgroup identified six specific areas related to COVID-19: Perinatal/Neonatal Transmission, Clinical Features of Neonatal Infection, Diagnostic and Serologic Testing, Management and Therapeutics, NICU-specific 
guidelines, and Consensus Statements and Reviews. Neonatologists self-identified with the topic areas, and two "topic experts" were assigned to each. Recognizing the significant lag time before COVID-related perinatal articles were indexed to PubMed, we generated a list of the major journal publishers (LWW/Wolters Kluwer, the AAP collection, Elsevier, the New England Journal of Medicine, JAMA network, BMJ, CDC, Springer, Lancet, Wiley, and Nature) and divided the journal publishers among the workgroup members. Every week, workgroup members surveyed their assigned publishers for new publications related to COVID-19 in pregnant women and infants. Finding such an article, each member used a standardized online form (Google Forms, Google Inc.) to abstract the citation, topic area, hyperlink and two to three bullet points summarizing the article's salient findings. Submission of a form automatically populated the article in a searchable, sortable, shareable database [1] (Google Drive, Google Inc.) that currently includes over 630 discrete publications. NeoCLEAR topic experts scanned their assigned publisher (s) for articles in any topic area to add to the database and reviewed in more depth all articles posted from their individual topic area. On average, NeoCLEAR team members spent $1 \mathrm{~h}$ per week updating the database and reviewing their topic area, and $1 \mathrm{~h}$ in the team meeting discussion.

\section{Dissemination}

We held weekly Zoom meetings with our section and neonatologists at affiliate institutions, during which the pairs of "topic experts" reviewed the most important publications from their topic area. Medical directors of our system's 5 affiliate level II and level III NICU's as well as neonatologists from across the region were invited to participate in the Zoom meetings. NeoCLEAR gained wider interest via mentions on the AAP Section on NeonatalPerinatal Medicine's Twitter account (@AAPNeonatal), as well as perinatalcovid19.org (maintained by Dr. Gautham Suresh, of Texas Children's Hospital). To-date, the NeoCLEAR database contains over 630 discrete articles related to neonatal-perinatal COVID-19, comprised of $\sim 60 \%$ peerreviewed articles, $25 \%$ guidelines and reviews, $10 \%$ letters to the editor and commentaries, and 5\% official webpages. It has had over 200 unique visitors and been accessed more than 2300 times. In order to spread the lessons learned from NeoCLEAR more widely, we prepared biweekly PowerPoint summaries of each topic area with hyperlinks to relevant publications. These are published on the AAP SoNPM's collaboration site [2], where they have been accessed by hundreds of neonatologists worldwide.

\section{Engagement and evolution}

As the pandemic wore on, guidelines from the CDC and American Academy of Pediatrics provided clarity and built consensus around some of the questions above. The NeoCLEAR group sought to broaden our initial objectives, maximize team member efficiency and maintain involvement as neonatologists in our section slowly resumed prepandemic research endeavors. And the neonatology community broadly pondered new questions:

- What is the impact of antenatal steroid therapy-a mainstay of modern perinatology — on mothers ill with COVID-19?

- Given the virus's penchant for endothelial derangements, what are the implications for pregnant women already at higher risk for thrombotic complications?

- Should our own personnel practices change, to protect senior physicians and pediatric trainees from unnecessary exposure?

Slowly, our group noted a shift in publication types from many single-center case reports and commentaries, to fewer but more rigorous analyses of multicenter, national and international registries (including the National Perinatal COVID-19 Registry (NPC-19), a joint effort by the AAP SoNPM and Vermont-Oxford Network.) NeoCLEAR "topic experts" shifted from surveying the literature for anything related to their assigned topic, to finding publications that would specifically address the new questions outlined above. We shifted to biweekly virtual meetings, with summary slides prepared for the AAP SoNPM through September 2020.

\section{Current state and future directions}

Beginning in November 2020, the United States has faced a spike in COVID-19 cases more dramatic and more pervasive than any thus far. Our group, like those who care for pregnant women and newborns across the country, ponders new questions:

- What story do SARS-CoV-19 antibody levels tell, and can we trust them?

- How do we protect both infants and NICU personnel from COVID-19, while supporting the NICU family and promoting parental bonding?

- What to make of the decrease in preterm birth seen in Scandinavian countries?

- As a vaccine becomes reality in the coming months, do the differences in the neonatal immune system have implications for COVID-19? 
There is still no playbook for a novel pandemic. But as the months have progressed, indexing via PubMed has "caught up" and publishers including the AAP, BMJ, and Lancet maintain their own virtual resources that compile the relevant Coronavirus literature. NeoCLEAR shifted to monthly meetings, attempting to address more nuanced questions and meet the needs of our regional NICU medical directors to inform changes in unit policies. We have also begun incorporating topics not strictly clinical: How can we fight burnout as the months of instability and fear drag on? How do we advocate for the academic neonatologists at most risk for childcare-related and career-related setbacks during the pandemic; namely, women in their early-and mid-careers? Thus, NeoCLEAR also became a social support network for our Neonatology section; a place to pose questions and share experiences as we are now necessarily isolated from one another. With time and the dissemination of an effective vaccine, we look forward to a decreased need for the NeoCLEAR team's comprehensive literature review efforts in 2021. We plan to continue periodic remote meetings and maintaining the database for the next six months. Waves of COVID-19 will likely continue to emerge along with new themes in the literature (importantly, the impact on mother-baby infrastructure in the developing world, and vaccine implications for pregnant and breastfeeding women.) The NeoCLEAR workgroup achieved its main objectives: to help neonatologists access and operationalize available evidence quickly, and adapt to changing information in an unstable, unprecedented time.

Acknowledgements With gratitude to Susan Niermeyer, MD, FAAP, James Barry, MD, FAAP and the NeoCLEAR team at the University of Colorado/Children's Hospital Colorado.

Funding CW is supported by NIH grant R01HL132941.

\section{Compliance with ethical standards}

Conflict of interest The authors declare no competing interests.

Publisher's note Springer Nature remains neutral with regard to jurisdictional claims in published maps and institutional affiliations.

\section{References}

1. NeoCLEAR Database Website. Open access. https://docs.google. com/spreadsheets/d/1L9tsrLn9a7LMql_nnUfMA3uS1SSurrj4XUh 2yT2bEUc/edit?usp=sharing. Last Accessed 16 Feb 2021.

2. AAP Collaboration Website, accessible with American Academy of Pediatrics (AAP) login. https://collaborate.aap.org/SONPM/Pages/ Perinatal\%20COVID-19.aspx. Accessed 16 Feb 2021. 